\title{
BMJ Open Development of a theoretical framework of factors affecting patient safety incident reporting: a theoretical review of the literature
}

\author{
Stephanie Archer, ${ }^{1}$ Louise Hull, ${ }^{1,2}$ Tayana Soukup, ${ }^{1}$ Erik Mayer, ${ }^{1}$ \\ Thanos Athanasiou, ${ }^{1}$ Nick Sevdalis, ${ }^{1,2}$ Ara Darzi ${ }^{1}$
}

To cite: Archer S, Hull L, Soukup T, et al. Development of a theoretical framework of factors affecting patient safety incident reporting: a theoretical review of the literature. BMJ Open 2017;7:e017155. doi:10.1136/ bmjopen-2017-017155

- Prepublication history and additional material for this paper are available online. To view these files, please visit the journal online (http://dx.doi. org/10.1136/bmjopen-2017017155).

Received 4 April 2017 Revised 24 July 2017 Accepted 2 August 2017

CrossMark

${ }^{1} \mathrm{NIHR}$ Imperial Patient Safety Translational Research Centre, Imperial College London,

London, UK

${ }^{2}$ Centre for Implementation Science, King's College London, London, UK

Correspondence to Dr Stephanie Archer; stephanie.archer@imperial. ac.uk

\section{ABSTRACT}

Objectives The development and implementation of incident reporting systems within healthcare continues to be a fundamental strategy to reduce preventable patient harm and improve the quality and safety of healthcare. We sought to identify factors contributing to patient safety incident reporting.

Design To facilitate improvements in incident reporting, a theoretical framework, encompassing factors that act as barriers and enablers ofreporting, was developed. Embase, Ovid MEDLINE(R) and PsycINF0 were searched to identify relevant articles published between January 1980 and May 2014. A comprehensive search strategy including MeSH terms and keywords was developed to identify relevant articles. Data were extracted by three independent researchers; to ensure the accuracy of data extraction, all studies eligible for inclusion were rescreened by two reviewers.

Results The literature search identified 3049 potentially eligible articles; of these, 110 articles, including $>29$ 726 participants, met the inclusion criteria. In total, 748 barriers were identified (frequency count) across the 110 articles. In comparison, 372 facilitators to incident reporting and 118 negative cases were identified. The top two barriers cited were fear of adverse consequences (161, representing $21.52 \%$ of barriers) and process and systems of reporting (110, representing $14.71 \%$ of barriers). In comparison, the top two facilitators were organisational (97, representing $26.08 \%$ of facilitators) and process and systems of reporting $(75$, representing $20.16 \%$ of facilitators).

Conclusion A wide range of factors contributing to engagement in incident reporting exist. Efforts that address the current tendency to under-report must consider the full range of factors in order to develop interventions as well as a strategic policy approach for improvement.

\section{BACKGROUND}

The development and implementation of incident reporting systems within healthcare continues to be a fundamental strategy to reduce preventable patient harm and improve the quality and safety of healthcare on a local,
Strengths and limitations of this study

- The synthesis included quantitative, qualitative and mixed methods research and was not restricted to specific incident reporting systems.

- Only articles published in English were included.

- The last systematic search for literature was conducted on 29 May 2014, meaning that literature published since this date will not have been included.

- Studies detailing interventions to improve incident reporting and studies detailing variations in engagement in incident reporting were not included.

- Large heterogeneity across studies in terms of outcome measures and methodologies meant conduction of meta-analysis was precluded.

regional and national basis. ${ }^{2}$ Although coverage and sophistication vary widely, incident reporting systems have now been in place for more than a decade in a number of countries. $^{3}$

A key factor that compromises the ability of incident reporting systems to improve patient safety is under-reporting. In the USA, it is estimated that $50 \%-96 \%$ of incidents are not reported. ${ }^{245}$ Failure to report patient safety incidents significantly hinders the underlying goals of incident reporting systems; low levels of reporting make it difficult at best to identify and prioritise patient safety risks and hamper learning from such incidents and ultimately improvements in patient safety. While debate continues to exist regarding whether all patient safety incidents should be reported, ${ }^{67}$ it is extremely important to understand the factors that act as barriers and facilitators to incident reporting so that 'sufficient' levels of reporting exist to facilitate learning and improvement.

A number of studies exploring barriers and facilitators to incident reporting have been conducted..$^{8-11}$ In addition, a number 
of literature reviews to identify barriers and facilitators to incident reporting have been published. ${ }^{12-14}$ Although previous work has made a valuable contribution to our understanding of factors affecting incident reporting, previous work has been limited in scope (eg, focusing on the psychological factors affecting incident reporting; ${ }^{14}$ focusing on perceived barriers influencing incident reporting by nurses $;{ }^{13}$ factors affecting reporting of incidents related to medical devices and other healthcare technologies). ${ }^{12}$ As such, to date, there has been no definitive synthesis and evaluation of the factors that prevent or promote reporting.

The primary aim of this theoretical review was to systematically identify factors affecting patient safety incident reporting. The secondary aims were, first, to develop a theoretical framework of factors acting as barriers and facilitators to incident reporting to guide implementation of interventions to increase engagement, and, second, to determine the prevalence of factors to guide the development of interventions and policies to improve incident reporting.

\section{METHODS}

\section{Theoretical review}

A theoretical review was conducted as the overarching goal of the review was to build explanation of factors affecting incident reporting. In line with a theoretical review, both quantitative and qualitative data were eligible for inclusion and interpretive methods were used to synthesise findings.

\section{Study searches and selection}

A systematic search strategy was developed and an electronic search was carried out in three databases: Embase, Ovid MEDLINE(R) and PsycINFO. The last search was conducted on 29 May 2014; while the last search was conducted 3 years ago, this reflects the sheer volume of articles that were included in this review. Search terms included those related to patient safety incidents, incident reporting systems, and barriers and facilitators to engagement in reporting (see table 1 for full search terms). Time and language of publications was restricted from 1980 and to English language.

\section{Eligibility criteria}

\section{Inclusion criteria}

1. Studies reporting factors influencing the likelihood of incident report engagement in any healthcare setting (eg, primary and secondary healthcare) and employing any study design (eg, qualitative, quantitative, mixed methods).

\section{Exclusion criteria}

1. Studies reporting aspects of incident reporting systems and/or incident reporting perceived positively and/or negatively by healthcare professionals (HCPs)

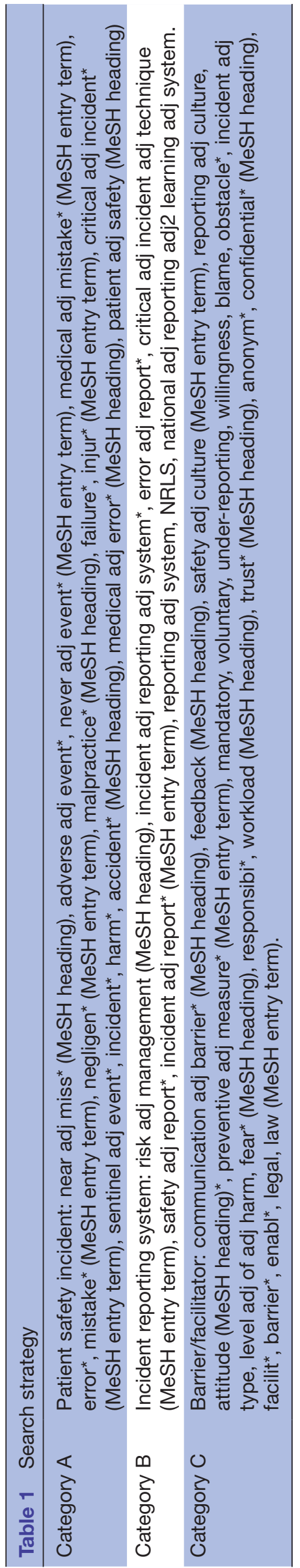


without data relating perceptions to incident reporting engagement.

2. Studies reporting data relating to disclosure of patient safety incidents to patients or their families (a systematic review of the literature on patient/family disclosure has previously been published). ${ }^{15}$

3. Studies reporting data relating to the effectiveness of interventions to improve incident reporting (a systematic review of the literature on the effectiveness of interventions to increase clinical incident reporting in healthcare has previously been published). ${ }^{13}$

4. Studies reporting statistical models where the impact of individual barriers and facilitators to engagement in incident reporting was unable to be determined.

The eligibility criteria were developed to maintain a focus on factors having a direct impact on incident reporting engagement rather than simply identifying and listing factors of incident reporting which were perceived positively or negatively by HCPs. Identifying elements of incident reporting perceived positively or negatively by HCPs does not equate to identifying factors that have an impact on reporting behaviour. In such studies, it is not possible to determine the impact on reporting behaviour-the primary focus of this review.

\section{Data extraction}

After the removal of duplicates, two authors (SA and $\mathrm{LH}$ ) independently reviewed all articles on the basis of the titles and abstracts. Three authors (SA, LH and TS) reviewed the articles at full-text stage. Data were extracted using an extraction template. The following data were extracted: first author's name, year of publication, country, study design, study population, sample size and factors that decrease (barriers), increase (facilitators) or were neither a barrier nor facilitator to engagement in incident reporting (negative cases). To ensure the accuracy of data extraction, all studies eligible for inclusion were rescreened by two reviewers (SA and LH).

\section{Quality assessment}

Many assessment tools and checklists have been developed to appraise the quality and susceptibility to bias of studies (eg, the Cochrane Collaboration's tool for assessing risk of bias in randomised trials; ${ }^{16}$ AMSTAR tool to assess the methodological quality of systematic reviews; ${ }^{17}$ tools to assess the quality of qualitative research studies). ${ }^{18}$ The decision not to assess the quality of studies was made for a number of reasons. First, the large heterogeneity of study designs would have made comparisons between study designs difficult at best. Second, quality appraisal is not considered necessary for theoretical reviews. ${ }^{19}$ Third, it has been argued that it is important, but difficult, to distinguish between 'quality of reporting' and the 'quality of a study'. ${ }^{20}$ As such, articles were not excluded from the current review based on 'quality' nor was weight assigned to studies based on quality.
Data analysis and initial theoretical framework development A grounded theory approach was used to guide the development of the theoretical framework. Grounded theory is associated with the discovery of theory from data systematically obtained from social research. ${ }^{21}$ It has been identified as a method where thorough and theoretically relevant analysis of a topic can be reached, specifically within literature reviews. ${ }^{22}$ In light of this, a three-stage approach was undertaken to develop a theory of factors contributing to engagement in patient safety incident reporting. The first stage, coding, includes identifying parts of the data that relate the phenomena in question (in this case, incident reporting). During this stage, known as open coding in the grounded theory literature, three authors (SA, LH and TS) read and re-read each paper and identified sections of the paper that were relevant to the research question. Initial concepts developed from these were noted down at this stage; in some cases, these were consistent with pre-existing literature (eg, in the case of a standardised scale), but in others allowed for unseen insights to develop across the data corpus (eg, in qualitative studies). In the second stage, conceptualising, or axial coding, focused on grouping together the initial codes where there were relationships to form higher-order categories. These were given names. Stage 3, categorising, or selective coding focused on linking together similar higher-order categories that contained similar concepts which could underpin the reasoning behind the way that the phenomena (in this case, incident reporting) could be explained. Figure 1 displays an example of how these stages were applied.

Engagement in these three stages allowed constant comparison between the articles in the data set to be performed until a theoretical framework was confirmed.

The final theoretical framework was reviewed by another member of the research team (NS) and feedback regarding the category descriptors was incorporated. The final theoretical framework of factors contributing to patient safety incident reporting engagement is displayed in table 2.

The theoretical framework developed was used to organise the identification of factors found to affect incident reporting and to quantify their prevalence. This approach is consistent with existing frameworks in the patient safety literature. For example, Lawton et al employed a similar approach to quantify the prevalence of factors contributing to patient safety incidents in hospital settings. ${ }^{23}$

\section{Patient and public involvement}

No patients were involved in setting the research question or the outcome measures, nor were they involved in the design and implementation of the study. We do not anticipate patients and the public being involved in the dissemination of the work.

\section{Findings}

The search identified 5335 records. After duplicates and limits were applied (English language, date restrictions 


\begin{tabular}{|c|c|c|c|}
\hline & $\begin{array}{c}\text { Stage 1: } \\
\text { Coding } \\
\text { (open coding) } \\
\text { ldentification of } \\
\text { data that } \\
\text { contributes to } \\
\text { engagement in } \\
\text { incident reporting }\end{array}$ & $\begin{array}{c}\text { Stage 2: } \\
\text { Conceptualising } \\
\text { (axial coding) } \\
\text { ldentification of } \\
\rightarrow \text { codes of similar } \\
\text { content grouped and } \\
\text { named }\end{array}$ & $\begin{array}{c}\text { Stage 3: } \\
\text { Categorising } \\
\text { (selective coding) } \\
\text { Identification of } \\
\rightarrow \text { similar concepts } \\
\text { grouped into broad } \\
\text { groups }\end{array}$ \\
\hline $\begin{array}{l}\text { Paper 1: } \\
\text { "Despite some } \\
\text { initial success with } \\
\text { this approach, } \\
\text { [implementation of } \\
\text { incident reporting } \\
\text { system] it was } \\
\text { stated that the time } \\
\text { and resource } \\
\text { demands of } \\
\text { managing the } \\
\text { process were too } \\
\text { great". }\end{array}$ & $\begin{array}{l}\text { Not enough time } \\
\text { Not enough } \\
\text { resources }\end{array}$ & $\rightarrow \quad \begin{array}{c}\text { Time } \\
\text { Resources }\end{array} \rightarrow$ & $\begin{array}{c}\text { Process and system } \\
\text { of reporting }\end{array}$ \\
\hline $\begin{array}{l}\text { Paper 2: } \\
\text { "The concept of a } \\
\text { blame culture } \\
\text { appeared to exist". }\end{array}$ & $\begin{array}{l}\text { People are scared } \\
\text { of being blamed }\end{array}$ & $\rightarrow$ Fear of blame & $\rightarrow \begin{array}{c}\text { Fear of adverse } \\
\text { consequences }\end{array}$ \\
\hline
\end{tabular}

Figure 1 Example of data coding, conceptualisation and categorisation for theory development.

1980-May 2014), 3049 records were considered for inclusion. Of these 3049 records, 2700 were excluded based on title and abstract screening. A total of 349 articles were considered potentially relevant and were assessed at fulltext by two researchers (kappa 0.70, p<0.001). Of 349 publications, 33 were not obtainable (requested through the British Library), leaving 314 articles assessed at fulltext stage. From these, 80 articles met inclusion criteria.

The reference lists of all included articles were screened for potentially relevant publications, resulting in a further 30 articles that met the inclusion criteria. A total of 110 articles, including $>29726$ participants, were included in the final review (figure 2). The total number of participants per study ranged from 8 to 2185 (mean=285.83; median: 134.00). Six studies did not report sample size, thus the sample size calculations represented above are based on 104 articles. ${ }^{24-29}$ See online supplementary table 1 for full data extraction.

\section{Study characteristics}

Empirical study types and design

In total, 110 articles were included; these consisted of 76 quantitative studies (including 72 questionnaire-based studies, 1 secondary analysis of data study, 1 case control study, 1 descriptive study and 1 cohort study), 21 qualitative studies (including 11 interview-based studies and 10 focus group studies) and 13 mixed-methods studies (1 semistructured interview and documentary analysis-based study; 1 semistructured interview and retrospective review of error reports-based study; 2 semistructured interview and questionnaire-based studies; 3 focus group and questionnaire-based studies; 1 semistructured and structured interview-based study; 1 interview, focus group and analysis of event reports-based study; 1 focus group and semistructured interview-based study; 1 retrospective analysis of routinely collected data and questionnaire-based study; 2 focus groups, interview and questionnaire-based studies).

\section{Countries}

The review encompassed research spanning 4 continents and $>20$ countries. The four countries contributing the most studies were the USA $(n=33)$, the UK $(n=24)$, Australia $(n=8)$ and Canada $(n=8)$, table 3.

\section{Year of publication}

A steady increase in articles was evident over decades: 1980s $\quad(\mathrm{n}=1),{ }^{30}$ 1990s $\quad(\mathrm{n}=12),{ }^{24} 31-41 \quad 2000 \mathrm{~s}$ $(\mathrm{n}=58),{ }^{8-11} 282942-93$ and 2010-May $2014(\mathrm{n}=39) .{ }^{25-2794-129}$ This increase is likely to reflect the growing integration of incident reporting systems in healthcare systems worldwide and the increasing realisation that HCPs' engagement in incident reporting is far from ideal.

The frequency of barriers and facilitators to incident reporting across the 110 articles was calculated and rank ordered across the data (figure 3). Where contributing factors were found not to be barriers or facilitators to incident reporting ( $\mathrm{eg}$, if fear was found not to be a 


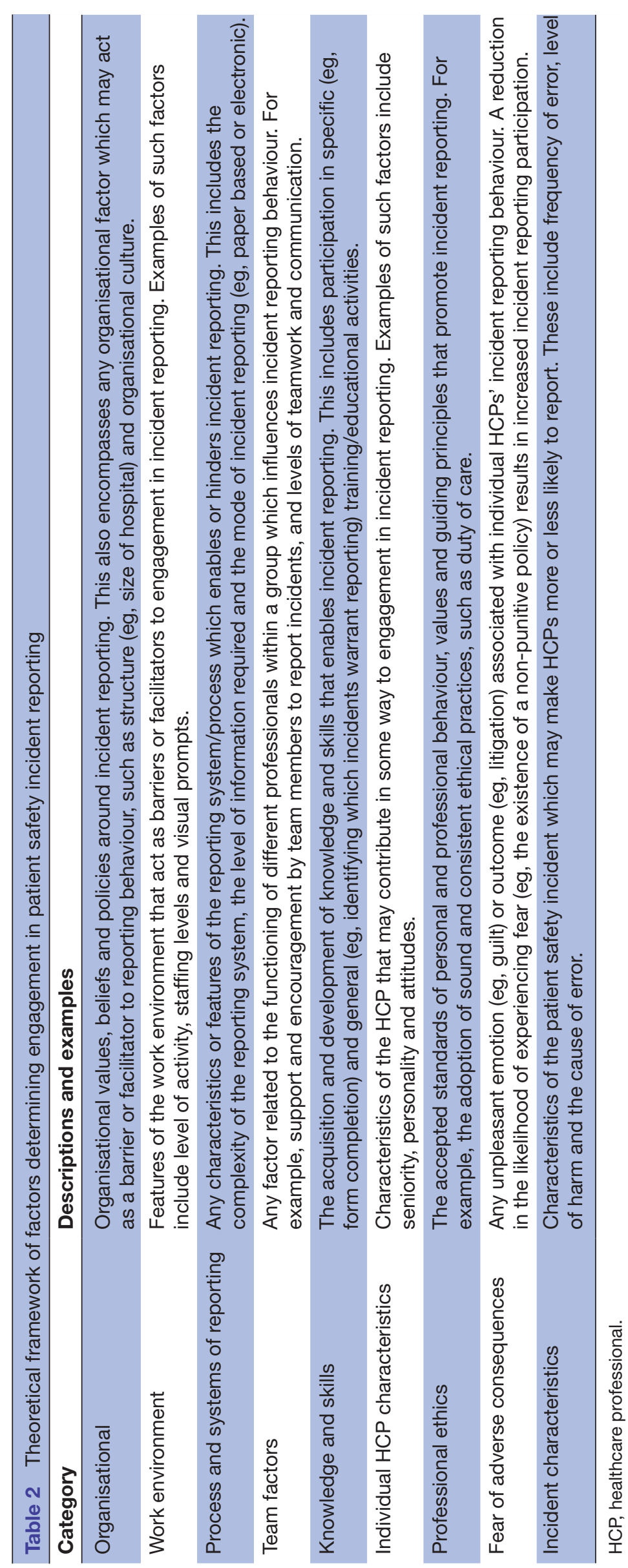




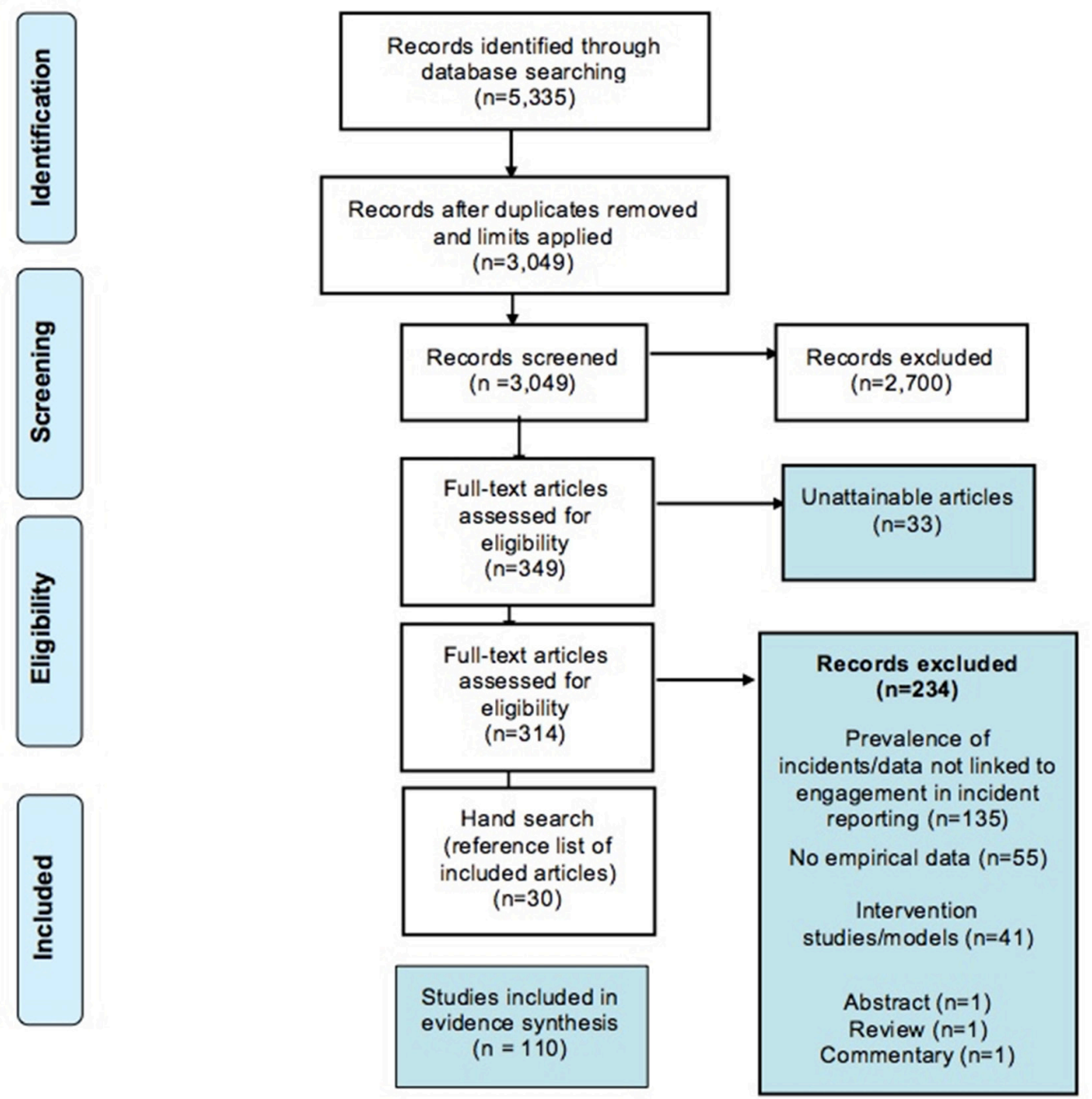

Figure 2 Flow diagram of the theoretical literature review process.

significant predictor of decreased or increased incident reporting), these were counted as negative cases. These negative cases were included to provide a more complete view of the data, and to prevent reporting bias.

When the same barrier, facilitator or negative case (eg, fear of adverse consequences) was mentioned more than once within an article, this was reflected in the frequency data presented. In total, 748 barriers to incident reporting were identified (frequency count) compared with 372 facilitators. A total of 118 negative cases were identified. The top two barriers cited were fear of adverse consequences (161, representing $21.52 \%$ of barriers) and process and systems of reporting (110, representing $14.71 \%$ of barriers). In comparison, the top two facilitators were organisational (97, representing $26.08 \%$ of facilitators) and process and systems of reporting (75, representing $20.16 \%$ of facilitators). These results illustrate that the factors identified in this review of the literature can act as both a barrier and a facilitator to incident reporting systems depending on context; for example, process and systems of reporting was found to be the second most frequently cited barrier, as well as the second most frequently cited facilitator to incident reporting engagement. While this may initially appear contradictory, when considering the complexity/simplicity of reporting it was found that highly complex incident reporting processes and systems were a barrier to incident reporting, whereas simple processes and systems were found to be a facilitator.

\section{Frequency of barriers to patient safety incident reporting}

Barriers to incident reporting were mentioned 748 times across the 110 articles (see online supplementary table $2)$. The three most frequently mentioned barriers to incident reporting included fear of adverse consequences $(161 / 748)$, process and systems of reporting $(110 / 748)$ and incident characteristics $(92 / 748)$.

\section{Fear of adverse consequences}

Fear of adverse consequences, as a barrier, was mentioned 161 times and included a general fear of adverse consequences associated with incident reporting $(51 / 161), 810$ 1127313339414244454748 51-53 59-61 63646971727679879498102 108109114116118120122126 fear of litigation $(30 / 161),^{8-1124}$ $2730323537-40444756677077808184869298108109116119129$ and 


\begin{tabular}{|c|c|}
\hline Country & Count (\%) \\
\hline USA $^{9112830-3342-6494-96}$ & $33(30.00)$ \\
\hline UK$^{10} 29$ 34-38 65-72 97-105 & $24(21.82)$ \\
\hline Australia 827397374 106-108 & $8(7.27)$ \\
\hline Canada $^{75-78}$ 109-112 & $8(7.27)$ \\
\hline Taiwan $^{79} 113-115$ & $4(3.64)$ \\
\hline Netherlands 4080116117 & $4(3.64)$ \\
\hline Saudi Arabia ${ }^{81} 118-120$ & $4(3.64)$ \\
\hline International $^{2426121} 122$ & $4(3.64)$ \\
\hline Israel $^{82} 83123$ & $3(2.73)$ \\
\hline $\operatorname{Iran}^{84124}$ & $2(1.82)$ \\
\hline$J_{a p a n}^{25} 125$ & $2(1.82)$ \\
\hline New Zealand 8586 & $2(1.82)$ \\
\hline Sweden 8788 & $2(1.82)$ \\
\hline Italy ${ }^{41126}$ & $2(1.82)$ \\
\hline Denmark $^{127}$ & $1(0.91)$ \\
\hline Norway $^{128}$ & $1(0.91)$ \\
\hline Pakistan ${ }^{129}$ & $1(0.91)$ \\
\hline Portugal $^{89}$ & $1(0.91)$ \\
\hline Jordan $^{90}$ & $1(0.91)$ \\
\hline China $^{91}$ & $1(0.91)$ \\
\hline Germany $^{92}$ & $1(0.91)$ \\
\hline Spain $^{93}$ & $1(0.91)$ \\
\hline
\end{tabular}

the fear of blame (24/161). $.^{8} 1035444752-54636471-737679$ 9798102103108111120 Additionally, the fear of judgement $(22 / 161),{ }^{1024343747525964727679818590109118122}$ the fear of the negative impact that incident reporting could have on relationships with other HCPs, patients and the public $(12 / 161),{ }^{10113353545664768594118126}$ and the fear of a detrimental impact that reporting an incident could have on

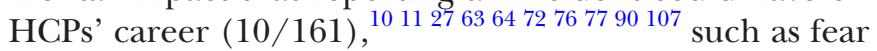
of job loss, were also cited as common barriers. Other less frequently mentioned barriers included protection of self (7/161), ${ }^{2436378191127}$ avoidance of discussion in meetings (4/161), ${ }^{86786108}$ and apprehension of sending an inappropriate form $(1 / 161) .{ }^{69}$

\section{Process and systems of reporting}

Process and systems of reporting was mentioned as a barrier to reporting 110 times. The most frequently identified barrier to incident reporting was the time required to complete an incident report $(29 / 110), 8112738394152$ $566267687172767779-81848795108109111116119120$

followed by the complexity of the reporting process $(28 / 110) .^{8911}$ 304345475354717277808186878995105 109-111 116119120 Other process and systems of reporting barriers included lack of anonymity and/or confidentiality in reporting $(22 / 110),{ }^{8}$ 1124273637475658687071808191102105108120 reporting format $(10 / 110), 394353737786111116$ and the type of reporting system (eg, paper-based) $(5 / 110) .{ }^{58768695}$ Less frequently mentioned barriers included lack of information to complete report $(3 / 110),{ }^{78} 8184$ the focus of reporting $(1 / 110),{ }^{71}$ and information to complete report not readily being available $(1 / 110){ }^{43}$

\section{Incident characteristics}

Incident characteristics were mentioned as a barrier to reporting 92 times. Level of harm, cause of incident and frequency of incident were the most frequent incident characteristics acting as barriers to reporting (40/92, 19/42 and 18/92, respectively). HCPs were less likely to report an incident if the patient experienced no or minimal harm. ${ }^{811} 2430313335373940434751-565859636667768490$ 9293101103105108109116119120122 Incidents that were deemed to occur frequently were considered too well-known to

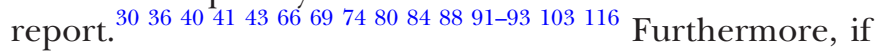
the cause of the incident was deemed unpreventable this acted as a barrier to incident reporting. 32 38-40 476673808184 889293116129 Other barriers included the type of incident $(13 / 92),{ }^{8} 3238394145466776778186116$ and the level of risk $(2 / 110) .^{1163}$

\section{Individual HCP characteristics}

Barriers reflective of individual HCP characteristics were cited 89 times. Barriers included a negative attitude/ lack of value placed on incident reporting $(53 / 89),{ }^{8} 936$ $3840414753546165666872767779-8186879298100102103105107-109$ 116119122126 and the perception that incident reporting does not result in improvements typically underlined such negative attitudes and values. A number of studies found that HCPs fail to report incidents because they simply forget $(9 / 89),{ }^{8} 27354377868893108$ and that the way HCPs perceive themselves can act as a barrier

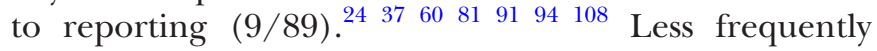
mentioned barriers included emotional responses to the incident (6/89), ${ }^{43} 637273116$ previous reporting behaviour $(5 / 89),{ }^{3246486897}$ exposure to errors (2/89), ${ }^{95114}$ and length of time in employment $(2 / 89) .^{48}$

\section{Knowledge and skills}

Knowledge and skills were cited as barriers to incident reporting 84 times. The two most frequently mentioned barriers related to a lack of reporting clarity $(36 / 84),{ }^{9} 11$ 2427303236374041434753547280818488919295103105108109116119

and a lack of clarity regarding what constitutes an adverse event and/or near miss (31/84). ${ }^{911} 303941434752-54676873$ 76777986108109112116119 This suggests that a lack of knowledge about what should be reported and how to do this act as barriers. Less frequently cited barriers included an inability in error recognition $(7 / 84),{ }^{4769727679120129}$ lack of training in reporting $(5 / 84), 3673102107114$ and lack of awareness $(4 / 84) .475284120$

\section{Work environment}

Work environment was mentioned 80 times as a barrier to incident reporting. Workload/priority (50/80), ${ }^{9} 112427$ 30 35-37 $4043464752565760-6367697073767786888991-9398102103106$ 109-111 116126 and accessibility (27/80), ,4 2730323741434647 6168697377808184868891107119120 were the most frequently 


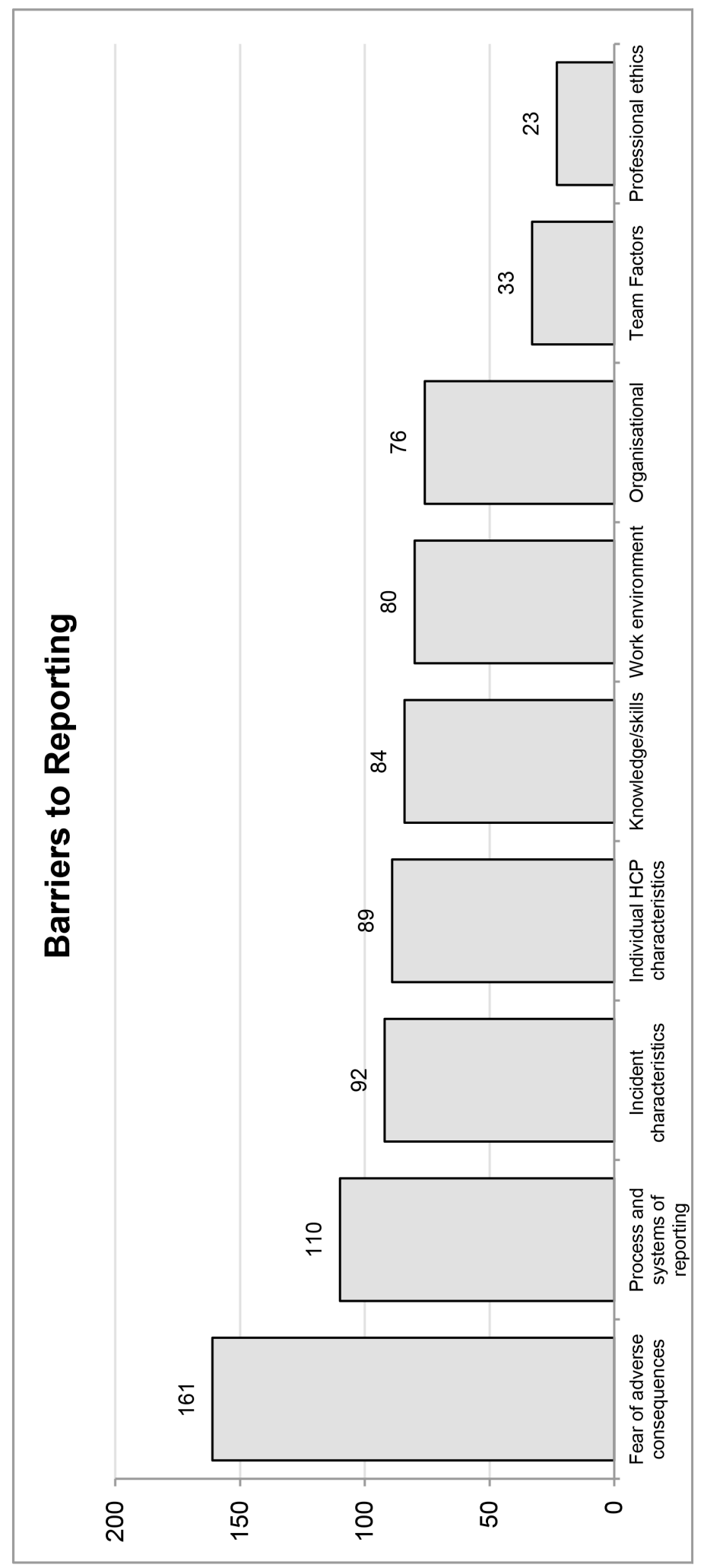

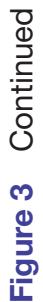




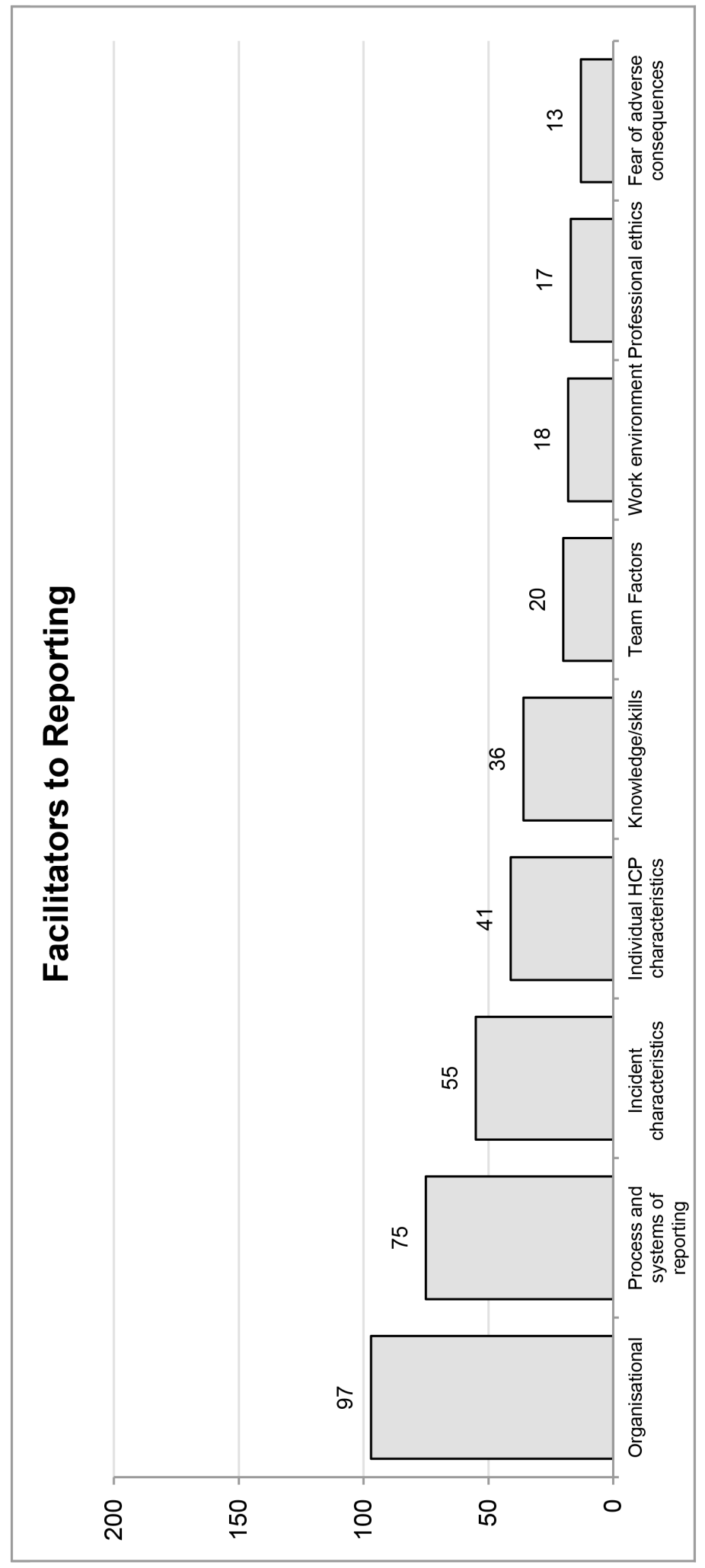

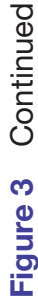




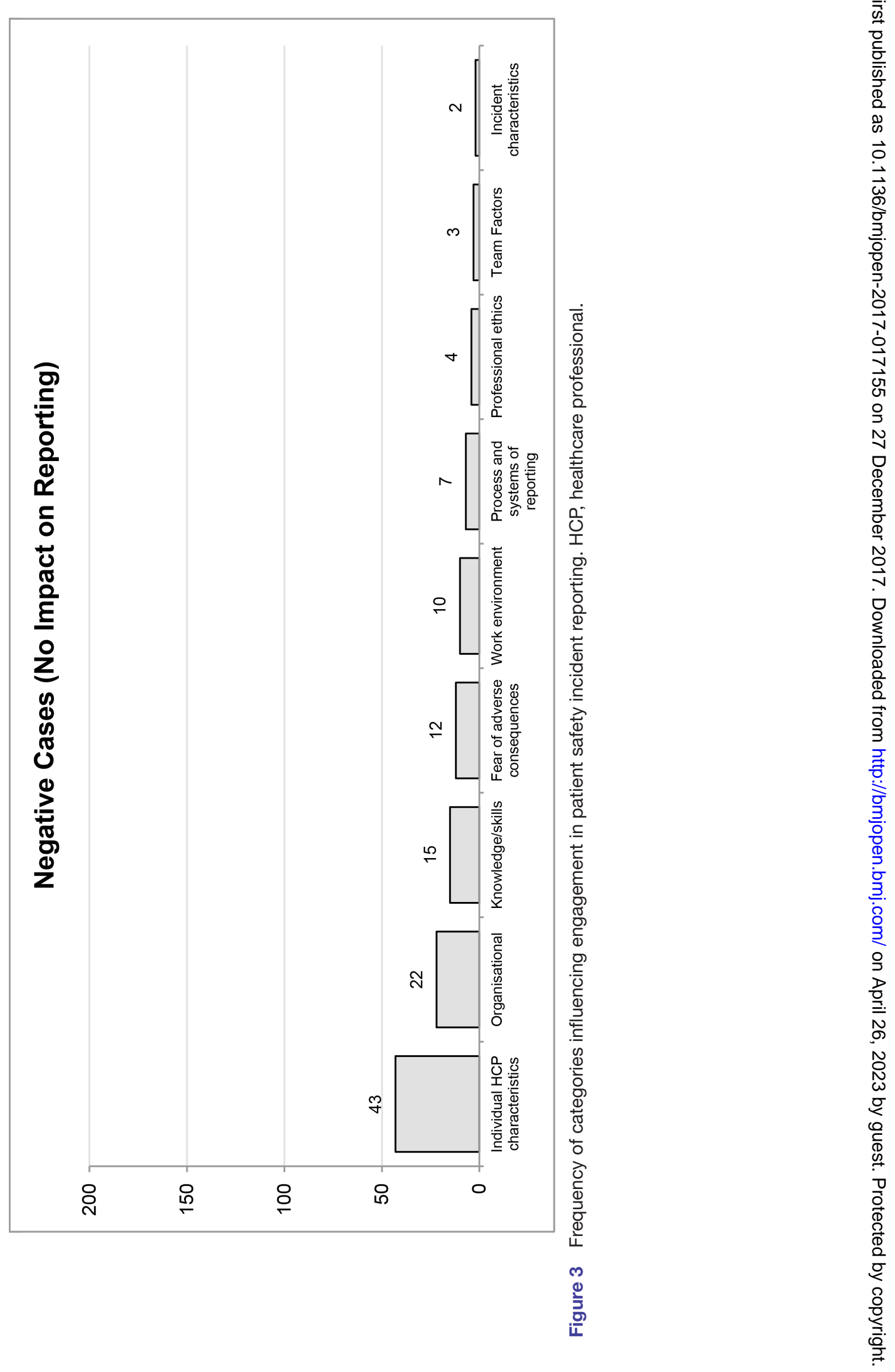


mentioned work environment barriers, suggesting that high workload does not allow for incident reporting to be prioritised, and that access to the reporting system is problematic (eg, not enough computer work stations to access reporting forms).

\section{Organisational factors}

Organisational factors were mentioned 76 times as a barrier to incident reporting. Lack of feedback and communication following incident reporting $(26 / 76),{ }^{89}$ 11394748525361636467717679869899107108111116120121128 and the absence/lack of a positive reporting culture $(17 / 76),{ }^{9} 1038$ 4647576676848687103107111128 were the two most frequently mentioned organisational barriers to reporting. Less frequently mentioned were lack of organisational learning and improvement $(7 / 76),{ }^{27} 39476798102116$ poor organisational use of data $(7 / 76),{ }^{52} 64767998$ and poor management response to reports $(5 / 76){ }^{60727683102}$

\section{Team factors}

Team factors were mentioned as barriers to engagement in incident reporting 33 times. The three most frequently mentioned barriers included the negative impact that incident reporting could have on working relationships $(13 / 33),{ }^{11274460636668108109111116}$ the influence of seniors

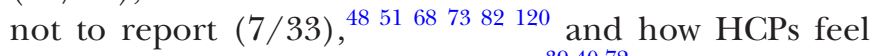
about reporting their peers $(5 / 33) .^{39} 4072$

\section{Professional ethics}

Professional ethics was the least frequently mentioned barrier to incident reporting (23/748). The most prevalent factor was a lack of personal responsibility to report $(15 / 23), 89324146475377788792103116118$ with studies suggesting that HCPs are less likely to report when they feel that reporting is the responsibility of someone else within the team. Concealment was also mentioned as a barrier $(5 / 23) .39108126$

\section{Frequency of facilitators in patient safety incident reporting}

Facilitators of reporting were mentioned 372 times across the 110 articles (see table 2). Organisational factors were the most frequently mentioned facilitator to incident reporting $(97 / 372)$, followed by process and systems of reporting (75/372) and incident characteristics (55/372) (online supplementary table 1 ).

\section{Organisational factors}

Organisational factors were mentioned as facilitators 97 times. The two most frequently cited facilitators included the provision of feedback/communication following incident reporting (29/97),, 1136424550535469708081838698 101-103 108116 and a non-punitive incident reporting policy (22/97). 91129363842444549546369708081102108120 The existence of a reporting culture (16/97), ${ }^{29} 41456669828396113116$ 120123127 and a focus on learning and improvement from incidents (13/97), ${ }^{9} 3943498298102103111116$ were also facilitators to reporting.

\section{Process and systems of reporting}

Process and systems of reporting was mentioned as a facilitator 75 times. Reporting format, ensuring anonymity and/or confidentiality, and simplification of reporting were the three most frequently cited facilitators accounting for $21 / 75,{ }^{9} 11254253546369818698101-103108116$ ${ }^{120} 16 / 75,{ }^{911} 294349536886101102108116120$ and $15 / 75,{ }^{9} 113842$ 70808695101102105116 facilitators within this category. Less frequently mentioned process and systems of reporting facilitators included the type of reporting system used (eg, electronic reporting) $(11 / 75) .^{454649} 538086102105$

\section{Incident characteristics}

Incident characteristics were mentioned as a facilitator to reporting 55 times. Level of harm and frequency of an incident were the most frequently cited incident characteristics identified as facilitators to reporting (26/55, 11394143495155586366697073848992109112129 and $13 / 55,{ }^{11} 4166697084129$ respectively). Incidents resulting in severe harm (including death) were more likely to be reported and HCPs were more likely to report incidents that occur infrequently rather than frequently. Less frequently mentioned facilitators included the type of incident $(8 / 55),{ }^{3941} 73$ cause of the incident $(6 / 55), 3649667089$ and level of risk $(1 / 55) .^{63}$

\section{Individual HCP characteristics}

Individual HCP characteristics were mentioned 41 times as a facilitator. A positive attitude towards incident reporting and a high value placed on incident reporting was found to increase the likelihood of reporting $(21 / 41) .{ }^{911} 496373$ 778189102109111112114115123 HCPs' emotional response to a patient safety incident was also found to increase the likelihood of reporting in a number of studies $(5 / 41){ }^{43} 63116$ The professional group of HCPs was also found to act as a facilitator to reporting $(5 / 41) \cdot{ }^{28} 104$ Less frequently cited facilitators included previous reporting behaviour $(1 / 41),{ }^{29}$ number of hours worked $(1 / 41),{ }^{32}$ and demographics (eg, gender and age) $(2 / 41) .{ }^{4815}$

\section{Knowledge and skills}

Training in reporting was identified as the most frequently mentioned facilitator in this category $(21 / 36) .{ }^{9} 253645$ 69808691103105108120 Other facilitators included knowledge regarding what constitutes an adverse event/near miss and the ability to recognise an error has occurred $\left(7 / 36,{ }^{9} 425354103108116\right.$ and 4/36, ${ }^{36} 6970129$ respectively).

\section{Team factors}

Team factors were mentioned 20 times as a facilitator to reporting. Good teamwork/communication $(7 / 20){ }^{69} 7096127$ and a positive team culture $(4 / 20),{ }^{81} 115123127$ were the most frequently cited facilitators.

\section{Professional ethics}

Professional ethics was cited as a facilitator 17 times. A strong sense of duty (8/17), ${ }^{39} 698081109112$ and responsibility $(5 / 17),,^{70757811}$ to report increased the likelihood 
of reporting. Less frequently cited facilitators included accountability $(2 / 17),{ }^{41} 109$ and a legal obligation to report $(1 / 17) .{ }^{48}$

\section{Work environment}

Work environment was mentioned as a facilitator 18 times. Access to the incident reporting system $(11 / 18),{ }^{42}$ 68698086102105108116 and those whose workloads allowed for and those that prioritised incident reporting increased the likelihood of reporting.

\section{Fear of adverse consequences}

Fear of adverse consequences was mentioned as a facilitator to reporting 13 times and included a fear of litigation and fear of blame increasing the likelihood of reporting $\left(8 / 13,{ }^{911} 274573109111\right.$ and 4/13, ${ }^{911} 108109$ respectively).

\section{Frequency of negative cases}

Negative cases were identified 118 times across the 110 articles (see table 2). The three most frequently mentioned factors included individual HCP characteristics (43/118), organisational factors $(22 / 118)$, and knowledge and skills (15/118), (online supplementary table 1).

Individual HCP characteristics were mentioned as a negative case 43 times. HCPs' attitude and value of incident reporting did not have an impact on reporting behaviour (12/43). ${ }^{33} 3548567293113$ Similarly, HCPs' demographics (eg, age, gender) had no impact on the likelihood of reporting (12/43). ${ }^{30} 324857708993113$ 114 Other less frequently mentioned factors included seniority $(4 / 43),{ }^{48} 708993$ forgetfulness $(1 / 43),{ }^{93}$ previous reporting behaviour $(1 / 43),{ }^{93}$ and number of hours worked $(1 / 43) .{ }^{26}$ Organisational factors were cited as having no impact on incident reporting 22 times. The most frequently mentioned were the ownership of the organisation (eg, private/public funded) $(6 / 22),{ }^{25} 70$ and management response towards incident reporting $(4 / 22) .{ }^{29} 114125$ Knowledge and skills were mentioned 15 times. These included the clarity of the reporting mechanism (5/15), ${ }^{29} 355693$ knowledge of what constitutes an adverse event/near miss $(2 / 15),{ }^{48,72}$ ability in error recognition $(1 / 15),{ }^{56}$ and training in error reporting $(7 / 15) .257093107$

Fear of adverse consequences was cited as having no impact on engagement in incident reporting 12 times. These included a fear of litigation $(4 / 12), 244956111$ a general fear of adverse consequences (3/12), ${ }^{35} 39113$ blame $(1 / 12),{ }^{56}$ judgement $(1 / 12),{ }^{80}$ and impact on career $(1 / 12) .{ }^{89}$ Work environment was mentioned as having no impact on reporting 10 times, including workload/ priority $(3 / 10),{ }^{30} 89128$ and unit type $\left.(3 / 10)\right)^{5783}$ Other less frequently cited work environment factors included physical work conditions $(1 / 10),{ }^{26}$ satisfaction with work environment $(1 / 10),{ }^{124}$ and accessibility $(1 / 10){ }^{56}$

Across all studies, process and systems of reporting was mentioned seven times as having no impact on incident reporting; these included reporting format $(3 / 7),{ }^{25} 89102$ complexity/simplification of reporting
$(1 / 7),{ }^{102}$ and anonymity and/or confidentiality $(1 / 7){ }^{24}$ Professional ethics were only mentioned four times as having no impact on the likelihood of incident reporting; these were legal obligation $(2 / 4),{ }^{48}$ duty $(1 / 4),{ }^{89}$ and responsibility $(1 / 4){ }^{26}$ Team factors were cited as having no impact on the likelihood of reporting three times, including teamwork and communication $(2 / 3),{ }^{128}$ and support/encouragement to report $(1 / 3) .{ }^{122}$ Incident characteristics were the least frequently mentioned factor which had no impact on reporting. Cause of incident was found to have no impact on engagement in reporting $(2 / 2){ }^{89} 93$

\section{DISCUSSION}

It has been suggested that there is a tendency in healthcare to encourage reporting of any and all patient safety incidents, to celebrate large quantities of incident reports and to aim for ever-increasing overall reporting rates. While there are numerous problems associated with this approach $^{7}$ (eg, flooding the system to such a degree that the thorough investigation of each incident reporting is unachievable), it is clear that high levels of under-reporting seriously compromise the ability of incident reporting systems to facilitate learning and improvements in patient safety.

This is the first theoretical literature review of factors contributing to patient safety incident reporting. Based on the evidence from 110 articles, we developed a theoretical framework, based on the principles of grounded theory, which summarises a wide range of factors contributing to incident reporting. We purposely sought publications from a range of countries, covering diverse health systems and study populations with a view to incorporating these into one broad theoretical framework. We argue that this is an appropriate approach for this initial explorative work, as multiple theoretical frameworks for individual counties, settings and populations (eg, nurses working in mental health settings in Australia), would have limited application at this point in time. However, we suggest that those interested in exploring barriers and facilitators in specific settings conduct further research using the theoretical framework presented here.

To improve incident reporting (both the quantity and/ or quality) and facilitate the successful implementation of incident reporting systems, we suggest that the theoretical framework is best used to prospectively and systematically identify factors within a given context that are likely to affect incident reporting. Those responsible for the effective implementation of incident reporting systems should explore each of the factors listed in our framework for salience. Rather than the framework being used in isolation, we recommend that it be used in conjunction with other implementation theories/frameworks and models to guide, understand and evaluate implementation of incident reporting systems. ${ }^{130}$ Based on such prospective analysis, strategies to enhance the adoption, implementation and sustainability of incident reporting systems can 
be tailored and selected according to a given setting. As such, using the developed framework will advance our understanding of how to optimally implement incident reporting systems into practice.

We used the developed theoretical framework, based on the evidence base, to organise our findings and have presented the frequency and rank order (ie, prevalence) of factors contributing to incident reporting. While this approach is consistent with other frameworks in the patient safety literature, ${ }^{1423}$ it may be considered as a crude analysis of the existing literature and needs to be interpreted with caution. We acknowledge that it is possible, although unlikely, that a relationship between the number of times a given factor is mentioned in the literature and its impact on incident reporting behaviour might not exist. However, we have been able to provide the first high-level overview of a large heterogeneous body of evidence. Furthermore, we acknowledge that weighting the impact of each factor would have been advantageous; however, the data did not lend itself to this possibility and we propose that it might not be possible to simply weight factors because of the complex and dynamic interrelationships that are likely to exist between them. Alternatively, we suggest that modelling the interrelationships between factors affecting incident reporting engagement is an avenue for future research.

Our results suggest that fear of adverse consequences and ineffective processes/systems of reporting are high-priority areas that require consideration to improve engagement in incident reporting. Changes to policy should be considered at an institutional or national level to prevent fear of litigation and blame, as fear of adverse consequences was found to inhibit incident reporting. We believe that it is unlikely that changes made within a single hospital or healthcare system would instil significant reassurance to promote incident reporting. In addition, at an organisational level we found that appropriate systems and processes for reporting need to be implemented to improve incident reporting; simultaneously, lack of or poorly designed systems significantly hinder reporting. These aspects of reporting rely on well-designed processes and technologies and are arguably the responsibility of organisational leaders. There is no 'optimum model' for incident reporting systems (eg, electronic, confidential, anonymous) - systems need to be responsive to users and organisational needs.

Organisational factors and processes/systems of reporting were identified as the two most frequently cited facilitators of reporting, which suggests that healthcare organisations consider these as high-priority areas which should be the target of increased focus and resources. For example, our results suggest that organisational policies that foster a reporting and learning culture as well as providing feedback following a report will promote incident reporting. Interestingly, we found that individual HCP characteristics have little impact on engagement in incident reporting. This suggests that organisations should be cautious before investing significant resources in these factors as such investment may result in minimal returns.

Although we have considered the above factors in isolation as illustrative examples, it is important to consider the interconnecting relationships between factors in order to develop intervention packages to improve engagement in incident reporting. Our results suggest that a comprehensive intervention/policy package which targets more than one contributing factor (eg, establishing a supportive work environment, with mechanisms which optimise shared learning, alongside a national policy to minimise the fear of adverse consequence) is far more likely to result in increased engagement in incident reporting compared with interventions that simply target one factor.

\section{Strengths and limitations}

In order to identify as much relevant literature as possible, we have included quantitative, qualitative and mixed-methods research and have not restricted the literature to specific incident reporting systems, that is, departmental, local, regional and national. In addition, the studies included a vast array of healthcare settings and providers, maximising the generalisability of the results. The resulting evidence has been synthetised into a practical output, that is, a theoretical framework to guide efforts to improve engagement in incident reporting.

The results and recommendations proposed in this evidence synthesis must be considered in light of several limitations. First, only articles published in English were included, which may generate bias. However, articles spanning 4 continents from $>20$ countries were identified, hence we are confident that our findings are of high external validity to guide safety policy globally. Second, the last systematic search for literature was conducted on 29 May 2014, meaning that literature published since this date will not have been included. We suggest that literature published after the last search could be useful to test the validity of the theoretical framework. Third, the decision not to include studies detailing interventions to improve incident reporting and studies detailing variations in engagement in incident reporting may skew the findings. This decision was made as it was not possible to determine the relative contribution of individual factors on engagement in incident reporting within such studies. Fourth, large heterogeneity across studies in terms of outcome measures and methodologies meant conduction of meta-analysis was precluded. This having been said, the synthesis of barriers and facilitators into frequency of reporting provides some evidence towards their respective relative importance, although it is accepted that the frequency of factors may represent those that have been the subject of more research. We recommend that future research applies and evaluates the usefulness of the developed theoretical framework in exploring and improving incident reporting in a variety of settings (eg, primary and secondary healthcare). 


\section{Future research}

There are many ways in which future research could test the validity of the theoretical framework presented in the current study. For example, content validity of the theoretical framework could be assessed using expert consensus methods (eg, Delphi study). In addition, predictive validity could be tested quantitatively by assessing the correlation between, for example, fear of adverse consequences (level of fear) and incident reporting behaviour (ie, number of incidents reported). A negative correlation between number of incidents reported (low) and fear of adverse consequence (high) would provide evidence for predictive validity of the theoretical framework.

\section{SUMMARY/CONCLUSION}

A wide range of factors contributing to engagement in incident reporting exist across varying levels of the healthcare system. Efforts aimed at addressing the current tendency to under-report must consider the full range of factors in order to develop tailored interventions and policy packages for improvement. We suggest the theoretical framework developed here would be useful in understanding factors affecting incident reporting engagement, increasing engagement in incident reporting and ultimately learning from patient safety incidents.

Acknowledgements The authors thank Kelsey Flott, BA, MSc, from the Centre for Health Policy, Imperial College London, for critically reviewing the manuscript.

Contributors Conception or design of the work: SA, LH, EM, NS and AD. Data collection: SA, LH and TS. Data analysis and interpretation: SA, LH, TA and NS. Drafting the article: SA, TA and NS. Critical revision of the article and final approval of the version to be published: SA, LH, TS, EM, TA, NS and AD.

Funding This article represents independent research supported by the National Institute for Health Research (NIHR) Imperial Patient Safety Translational Research Centre. LH and NS' research was supported by the National Institute for Health Research (NIHR) Collaboration for Leadership in Applied Health Research and Care (CLAHRC) South London at King's College Hospital NHS Foundation Trust. LH and NS are members of King's Improvement Science, which is part of the NIHR CLAHRC South London and comprises a specialist team of improvement scientists and senior researchers based at King's College London. Its work is funded by King's Health Partners (Guy's and St Thomas' NHS Foundation Trust, King's College Hospital NHS Foundation Trust, King's College London and South London and Maudsley NHS Foundation Trust), Guy's and St Thomas' Charity, the Maudsley Charity and the Health Foundation.

Disclaimer The views expressed are those of the author(s) and not necessarily those of the NHS, the NIHR or the Department of Health.

Competing interests None declared.

Provenance and peer review Not commissioned; externally peer reviewed.

Data sharing statement All data from this review and theoretical framework are presented within the publication.

Open Access This is an Open Access article distributed in accordance with the terms of the Creative Commons Attribution (CC BY 4.0) license, which permits others to distribute, remix, adapt and build upon this work, for commercial use, provided the original work is properly cited. See: http://creativecommons.org/ licenses/by/4.0/

(C) Article author(s) (or their employer(s) unless otherwise stated in the text of the article) 2017. All rights reserved. No commercial use is permitted unless otherwise expressly granted.
REFERENCES

1. Barach P, Small SD. Reporting and preventing medical mishaps: lessons from non-medical near miss reporting systems. BMJ 2000;320:759-63.

2. Leape LL. Error in medicine. JAMA 1994;272:1851-7.

3. Department of Health. An organisation with a memory: report of an expert group on learning from adverse events in the NHS. https:// psnet.ahrq.gov/resources/resource/1568 (accessed Mar 2017).

4. Noble DJ, Pronovost PJ. Underreporting of patient safety incidents reduces health care's ability to quantify and accurately measure harm reduction. J Patient Saf 2010;6:247-50.

5. Institute of Medicine. To err is human: building a safety health system. Washington, DC: National Academy Press, 1999.

6. Howell AM, Burns EM, Hull L, et al. International recommendations for national patient safety incident reporting systems: an expert Delphi consensus-building process. BMJ Qual Saf 2017;26.

7. Macrae C. The problem with incident reporting. BMJ Qual Saf 2016;25:71-5.

8. Evans SM, Berry JG, Smith BJ, et al. Attitudes and barriers to incident reporting: a collaborative hospital study. Qual Saf Health Care 2006;15:39-43.

9. Kingston MJ, Evans SM, Smith BJ, et al. Attitudes of doctors and nurses towards incident reporting: a qualitative analysis. Med $J$ Aust 2004;181:36-9.

10. Waring JJ. Beyond blame: cultural barriers to medical incident reporting. Soc Sci Med 2005;60:1927-35.

11. Jeffe DB, Dunagan WC, Garbutt J, et al. Using focus groups to understand physicians' and nurses' perspectives on error reporting in hospitals. Jt Comm J Qual Saf 2004;30:471-9.

12. Polisena J, Gagliardi A, Urbach D, et al. Factors that influence the recognition, reporting and resolution of incidents related to medical devices and other healthcare technologies: a systematic review. Syst Rev 2015;4:37.

13. Fung WM, Koh SS, Chow YL. Attitudes and perceived barriers influencing incident reporting by nurses and their correlation with reported incidents: A systematic review. JBI Libr Syst Rev 2012;10:1-65.

14. Pfeiffer $\mathrm{Y}$, Manser T, Wehner T. Conceptualising barriers to incident reporting: a psychological framework. Qual Saf Health Care 2010;19:e60.

15. O'Connor E, Coates HM, Yardley IE, et al. Disclosure of patient safety incidents: a comprehensive review. Int J Qual Health Care 2010;22:371-9.

16. Higgins JP, Altman DG, Gøtzsche PC, et al. The Cochrane Collaboration's tool for assessing risk of bias in randomised trials. BMJ 2011;343:d5928.

17. Shea BJ, Grimshaw JM, Wells GA, et al. Development of AMSTAR: a measurement tool to assess the methodological quality of systematic reviews. BMC Med Res Methodol 2007;7:10.

18. Long AF, Godfrey M. An evaluation tool to assess the quality of qualitative research studies. Int J Soc Res Methodol 2004;7:181-96.

19. Paré G, Trudel M-C, Jaana M, et al. Synthesizing information systems knowledge: A typology of literature reviews. Inf Manage 2015;52:183-99.

20. Sanderson S, Tatt ID, Higgins JP. Tools for assessing quality and susceptibility to bias in observational studies in epidemiology: a systematic review and annotated bibliography. Int $J$ Epidemiol 2007;36:666-76.

21. Glaser B, Strauss A. The Discovery of Grounded Theory. Hawthorne, New York: Aldine Publishing Company, 1967.

22. Wolfswinkel JF, Furtmueller E, Wilderom CPM. Using grounded theory as a method for rigorously reviewing literature. Eur J Inf Syst 2013;22:45-55.

23. Lawton R, McEachan RR, Giles SJ, et al. Development of an evidence-based framework of factors contributing to patient safety incidents in hospital settings: a systematic review. BMJ Qual Saf 2012;21:369-80.

24. Belton KJ. Attitude survey of adverse drug-reaction reporting by health care professionals across the European Union. Eur J Clin Pharmacol 1997;52:423-7.

25. Fukuda $\mathrm{H}$, Imanaka $\mathrm{Y}$, Hirose $\mathrm{M}$, et al. Impact of system-level activities and reporting design on the number of incident reports for patient safety. Qual Saf Health Care 2010;19:122-7.

26. Gaal S, van den Hombergh $P$, Verstappen W, et al. Patient safety features are more present in larger primary care practices. Health Policy 2010;97:87-91.

27. Jennings PA, Stella J. Barriers to incident notification in a regional prehospital setting. Emerg Med J 2011;28:526-9.

28. Rowin EJ, Lucier D, Pauker SG, et al. Does error and adverse event reporting by physicians and nurses differ? Jt Comm J Qual Patient Saf 2008;34:537-45. 
29. Hutchinson A, Young TA, Cooper KL, et al. Trends in healthcare incident reporting and relationship to safety and quality data in acute hospitals: results from the National Reporting and Learning System. Qual Saf Health Care 2009;18:5-10.

30. Rogers AS, Israel E, Smith CR, et al. Physician knowledge, attitudes, and behavior related to reporting adverse drug events. Arch Intern Med 1988;148:1596-600.

31. Schulmeister L. Chemotherapy medication errors: descriptions, severity, and contributing factors. Oncol Nurs Forum 1999;26:1033-42.

32. Generali JA, Danish MA, Rosenbaum SE. Knowledge of and attitudes about adverse drug reaction reporting among Rhode Island pharmacists. Ann Pharmacother 1995;29:365-9.

33. Osborne J, Blais K, Hayes JS. Nurses' perceptions: when is it a medication error? J Nurs Adm 1999;29:33-8.

34. Gladstone J. Drug administration errors: a study into the factors underlying the occurrence and reporting of drug errors in a district general hospital. J Adv Nurs 1995;22:628-37.

35. Vincent C, Stanhope N, Crowley-Murphy M. Reasons for not reporting adverse incidents: an empirical study. J Eval Clin Pract 1999;5:13-21.

36. Green CF, Mottram DR, Raval D, et al. Community pharmacists' attitudes to adverse drug reaction reporting. Int $J$ Pharm Pract 1999;7:92-9.

37. Belton KJ, Lewis SC, Payne S, et al. Attitudinal survey of adverse drug reaction reporting by medical practitioners in the United Kingdom. Br J Clin Pharmacol 1995;39:223-6.

38. Bateman DN, Sanders GL, Rawlins MD. Attitudes to adverse drug reaction reporting in the Northern Region. Br J Clin Pharmacol 1992;34:421-6.

39. Walker SB, Lowe MJ. Nurses' views on reporting medication incidents. Int J Nurs Pract 1998;4:97-102.

40. Eland IA, Belton KJ, van Grootheest AC, et al. Attitudinal survey of voluntary reporting of adverse drug reactions. $\mathrm{Br} J$ Clin Pharmacol 1999;48:623-7.

41. Cosentino M, Leoni O, Banfi F, et al. Attitudes to adverse drug reaction reporting by medical practitioners in a Northern Italian district. Pharmacol Res 1997;35:85-8.

42. Beasley JW, Escoto KH, Karsh BT. Design elements for a primary care medical reporting system. WMJ 2004;103:56-9.

43. Elder NC, Graham D, Brandt E, et al. Barriers and motivators for making error reports from family medicine offices: a report from the American Academy of Family Physicians National Research Network (AAFP NRN). J Am Board Fam Med 2007;20:115-23.

44. Fairbanks RJ, Crittenden CN, O'Gara KG, et al. Emergency medical services provider perceptions of the nature of adverse events and near-misses in out-of-hospital care: an ethnographic view. Acad Emerg Med 2008;15:633-40.

45. Karsh BT, Escoto KH, Beasley JW, et al. Toward a theoretical approach to medical error reporting system research and design. Appl Ergon 2006;37:283-95.

46. Kennedy AG, Littenberg B. Medication error reporting by community pharmacists in Vermont. J Am Pharm Assoc 2004;44:434-8.

47. Handler SM, Perera S, Olshansky EF, et al. Identifying modifiable barriers to medication error reporting in the nursing home setting. J Am Med Dir Assoc 2007;8:568-74.

48. Daly JM, Jogerst GJ. Association of knowledge of adult protective services legislation with rates of reporting of abuse in lowa nursing homes. J Am Med Dir Assoc 2005;6:113-20.

49. Garbutt J, Brownstein DR, Klein EJ, et al. Reporting and disclosing medical errors: pediatricians' attitudes and behaviors. Arch Pediatr Adolesc Med 2007;161:179-85.

50. Kaldjian LC, Jones EW, Wu BJ, et al. Reporting medical errors to improve patient safety: a survey of physicians in teaching hospitals. Arch Intern Med 2008;168:40-6.

51. Hohenhaus SM. Emergency nursing and medical error-a survey of two states. J Emerg Nurs 2008;34:20-5.

52. Patrician PA, Brosch LR. Medication error reporting and the work environment in a military setting. J Nurs Care Qual 2009;24:277-86.

53. Schectman JM, Plews-Ogan ML. Physician perception of hospital safety and barriers to incident reporting. Jt Comm J Qual Patient Saf 2006;32:337-43.

54. Taylor JA, Brownstein D, Christakis DA, et al. Use of incident reports by physicians and nurses to document medical errors in pediatric patients. Pediatrics 2004;114:729-35.

55. Throckmorton T, Etchegaray J. Factors affecting incident reporting by registered nurses: the relationship of perceptions of the environment for reporting errors, knowledge of the nursing practice act, and demographics on intent to report errors. J Perianesth Nurs 2007;22:400-12.
56. Uribe CL, Schweikhart SB, Pathak DS, et al. Perceived barriers to medical-error reporting: an exploratory investigation. J Healthc Manag 2002;47:263-79.

57. Vogus TJ, Sutcliffe KM. The impact of safety organizing, trusted leadership, and care pathways on reported medication errors in hospital nursing units. Med Care 2007;45:997-1002.

58. Weissman JS, Annas CL, Epstein AM, et al. Error reporting and disclosure systems: views from hospital leaders. JAMA 2005;293:1359-66.

59. Mayo AM, Duncan D. Nurse perceptions of medication errors: what we need to know for patient safety. J Nurs Care Qual 2004;19:209-17.

60. Blegen MA, Vaughn T, Pepper G, et al. Patient and staff safety: voluntary reporting. Am J Med Qual 2004;19:67-74.

61. King ES, Moyer DV, Couturie MJ, et al. Getting doctors to report medical errors: project DISCLOSE. Jt Comm J Qual Patient Saf 2006;32:382-92.

62. Coley KC, Pringle JL, Weber RJ, et al. Perceived barriers in using a region-wide medication error reporting system. J Patient Saf 2006;2:39-44

63. Elder NC, Brungs SM, Nagy M, et al. Nurses' perceptions of error communication and reporting in the intensive care unit. $J$ Patient Saf 2008;4:162-8.

64. Stratton KM, Blegen MA, Pepper G, et al. Reporting of medication errors by pediatric nurses. J Pediatr Nurs 2004;19:385-92.

65. Waring JJ. A qualitative study of the intra-hospital variations in incident reporting. Int J Qual Health Care 2004;16:347-52.

66. Ashcroft DM, Morecroft C, Parker D, et al. Likelihood of reporting adverse events in community pharmacy: an experimental study. Qual Saf Health Care 2006;15:48-52.

67. Kreckler S, Catchpole K, McCulloch P, et al. Factors influencing incident reporting in surgical care. Qual Saf Health Care 2009;18:116-20.

68. Sharma A, Jain P, Parmar B, et al. Incident Reporting in Surgical Trainees-Revisited. J Patient Saf 2008;4:191-4.

69. Green CF, Mottram DR, Rowe PH, et al. Attitudes and knowledge of hospital pharmacists to adverse drug reaction reporting. $\mathrm{Br} J \mathrm{Clin}$ Pharmacol 2001;51:81-6.

70. Sweis D, Wong IC. A survey on factors that could affect adverse drug reaction reporting according to hospital pharmacists in Great Britain. Drug Saf 2000;23:165-72.

71. McArdle D, Burns N, Ireland A. Attitudes and beliefs of doctors towards medication error reporting. Int $J$ Health Care Qual Assur 2003;16:326-33.

72. Sanghera IS, Franklin BD, Dhillon S. The attitudes and beliefs of healthcare professionals on the causes and reporting of medication errors in a UK Intensive care unit. Anaesthesia 2007;62:53-61.

73. Haines TP, Cornwell P, Fleming J, et al. Documentation of inhospital falls on incident reports: qualitative investigation of an imperfect process. BMC Health Serv Res 2008;8:254.

74. Johnstone MJ, Kanitsaki O. Patient safety and the integration of graduate nurses into effective organizational clinical risk management systems and processes: an Australian study. Qual Manag Health Care 2008;17:162-73.

75. Espin S, Levinson W, Regehr G, et al. Error or "act of God"? A study of patients' and operating room team members' perceptions of error definition, reporting, and disclosure. Surgery 2006;139:6-14

76. Covell CL, Ritchie JA. Nurses' responses to medication errors: suggestions for the development of organizational strategies to improve reporting. J Nurs Care Qual 2009;24:287-97.

77. Merchant RN, Gully PM. A survey of British Columbia anesthesiologists on a provincial critical incident reporting program. Can J Anaesth 2005;52:680-4.

78. Espin S, Regehr G, Levinson W, et al. Factors influencing perioperative nurses' error reporting preferences. Aorn $J$ 2007;85:527-43.

79. Chiang HY, Pepper GA. Barriers to nurses' reporting of medication administration errors in Taiwan. J Nurs Scholarsh 2006;38:392-9.

80. Mes K, Berg LTWdeJ-van, Grootheest AC. Attitudes of community pharmacists in the Netherlands towards adverse drug reaction reporting. Int J Pharm Pract 2002;10:267-72.

81. Bawazir SA. Attitude of community pharmacists in Saudi Arabia towards adverse drug reaction reporting. Saudi Pharm J 2006;14:75-83.

82. Kagan I, Barnoy S. Factors associated with reporting of medication errors by Israeli nurses. J Nurs Care Qual 2008;23:353-61.

83. Naveh E, Katz-Navon T, Stern Z. Readiness to report medical treatment errors: the effects of safety procedures, safety information, and priority of safety. Med Care 2006;44:2117-23. 
84. Vessal G, Mardani Z, Mollai M. Knowledge, attitudes, and perceptions of pharmacists to adverse drug reaction reporting in Iran. Pharm World Sci 2009;31:183-7.

85. Soleimani F. Learning from mistakes in New Zealand hospitals: what else do we need besides "no-fault"? N Z Med J 2006;119:U2099.

86. Yong $\mathrm{H}$, Kluger MT. Incident reporting in anaesthesia: a survey of practice in New Zealand. Anaesth Intensive Care 2003;31:555-9.

87. Söderberg J, Grankvist K, Brulin C, et al. Incident reporting practices in the preanalytical phase: Low reported frequencies in the primary health care setting. Scand J Clin Lab Invest 2009;69:731-5.

88. Bäckström M, Mjörndal T, Dahlqvist R, et al. Attitudes to reporting adverse drug reactions in northern Sweden. Eur J Clin Pharmacol 2000;56:729-32.

89. Herdeiro MT, Figueiras A, Polónia J, et al. Influence of Pharmacists' Attitudes on Adverse Drug Reaction Reporting. Drug Saf 2006;29:331-40.

90. Mrayyan MT, Shishani K, Al-Faouri I. Rate, causes and reporting of medication errors in Jordan: nurses' perspectives. J Nurs Manag 2007;15:659-70.

91. Li Q, Zhang SM, Chen HT, et al. Awareness and attitudes of healthcare professionals in Wuhan, China to the reporting of adverse drug reactions. Chin Med J 2004;117:856-61.

92. Hasford J, Goettler M, Munter KH, et al. Physicians' knowledge and attitudes regarding the spontaneous reporting system for adverse drug reactions. J Clin Epidemiol 2002;55:945-50.

93. Irujo M, Beitia G, Bes-Rastrollo M, et al. Factors that influence under-reporting of suspected adverse drug reactions among community pharmacists in a Spanish region. Drug Saf 2007;30:1073-82.

94. Church JA, Adams RD, Hendrix LH, et al. National study to determine the comfort levels of radiation therapists and medical dosimetrists to report errors. Pract Radiat Oncol 2013;3:e165-70.

95. Ehrenpreis ED, Sifuentes H, Ehrenpreis JE, et al. Suboptimal reporting of adverse medical events to the FDA Adverse Events Reporting System by nurse practitioners and physician assistants. Expert Opin Drug Saf 2012;11:177-83.

96. Erler C, Edwards NE, Ritchey S, et al. Perceived patient safety culture in a critical care transport program. Air Med $J$ 2013;32:208-15.

97. Anderson JE, Kodate N, Walters R, et al. Can incident reporting improve safety? Healthcare practitioners' views of the effectiveness of incident reporting. Int J Qual Health Care 2013;25:141-50.

98. Arfanis K, Smith A. Informal risk assessment strategies in health care staff: an unrecognized source of resilience? J Eval Clin Pract 2012;18:1140-6.

99. Armitage G, Newell R, Wright J. Improving the quality of drug error reporting. J Eval Clin Pract 2010;16:1189-97.

100. Turner S, Ramsay A, Fulop N. The role of professional communities in governing patient safety. J Health Organ Manag 2013;27:527-43.

101. Williams SD, Phipps DL, Ashcroft DM. Understanding the attitudes of hospital pharmacists to reporting medication incidents: a qualitative study. Res Social Adm Pharm 2013:9:80-9.

102. Holmström AR, Airaksinen M, Weiss M, et al. National and local medication error reporting systems: a survey of practices in 16 countries. J Patient Saf 2012;8:165-76.

103. Parvizi N, Robertson I, McWilliams RG. Medical device adverse incident reporting in interventional radiology. Clin Radiol 2014;69:263-7.

104. Sarvadikar A, Prescott G, Williams D. Attitudes to reporting medication error among differing healthcare professionals. Eur $J$ Clin Pharmacol 2010;66:843-53.

105. Winchester SA, Tomkins S, Cliffe S, et al. Healthcare workers' perceptions of occupational exposure to blood-borne viruses and reporting barriers: a questionnaire-based study. J Hosp Infect 2012;82:36-9.

106. Tariq A, Georgiou A, Westbrook J. Medication incident reporting in residential aged care facilities: limitations and risks to residents safety. BMC Geriatr 2012;12:67.

107. Braithwaite J, Westbrook MT, Travaglia JF, et al. Cultural and associated enablers of, and barriers to, adverse incident reporting. Qual Saf Health Care 2010;19:229-33.
108. Heard GC, Sanderson PM, Thomas RD. Barriers to adverse event and error reporting in anesthesia. Anesth Analg 2012;114:604-14.

109. Hartnell N, MacKinnon N, Sketris I, et al. Identifying, understanding and overcoming barriers to medication error reporting in hospitals: a focus group study. BMJ Qual Saf 2012;21:361-8.

110. Walji R, Boon H, Barnes J, et al. Reporting natural health product related adverse drug reactions: is it the pharmacist's responsibility? Int J Pharm Pract 2011;19:383-91.

111. Waters NF, Hall WA, Brown $\mathrm{H}$, et al. Perceptions of Canadian labour and delivery nurses about incident reporting: a qualitative descriptive focus group study. Int J Nurs Stud 2012;49:811-21.

112. Espin S, Wickson-Griffiths A, Wilson M, et al. To report or not to report: a descriptive study exploring ICU nurses' perceptions of error and error reporting. Intensive Crit Care Nurs 2010;26:1-9.

113. Chang IC, Hsu HM. Predicting medical staff intention to use an online reporting system with modified unified theory of acceptance and use of technology. Telemed J E Health 2012;18:67-73.

114. Chiang HY, Lin SY, Hsu SC, et al. Factors determining hospital nurses' failures in reporting medication errors in Taiwan. Nurs Outlook 2010;58:17-25.

115. Chiang HY, Lin SY, Hsiao YC, et al. Culture influence and predictors for behavioral involvement in patient safety among hospital nurses in Taiwan. J Nurs Care Qual 2012;27:359-67.

116. Martowirono K, Jansma JD, van Luijk SJ, et al. Possible solutions for barriers in incident reporting by residents. J Eval Clin Pract 2012;18:76-81.

117. Zwart DL, Heddema WS, Vermeulen MI, et al. Lessons learnt from incidents reported by postgraduate trainees in Dutch general practice. A prospective cohort study. BMJ Qual Saf 2011;20:857-62.

118. Alsafi E, Bahroon SA, Tamim H, et al. Physicians' attitudes toward reporting medical errors-an observational study at a general hospital in Saudi Arabia. J Patient Saf 2011;7:143-7.

119. Khan TM. Community pharmacists' knowledge and perceptions about adverse drug reactions and barriers towards their reporting in Eastern region, Alahsa, Saudi Arabia. Ther Adv Drug Saf 2013;4:45-51.

120. Tobaiqy M, Stewart D. Exploring health professionals' experiences of medication errors in Saudi Arabia. Int J Clin Pharm 2013;35:542-5.

121. Davies EC, Chandler $\mathrm{Cl}$, Innocent SH, et al. Designing adverse event forms for real-world reporting: participatory research in Uganda. PLoS One 2012;7:e32704.

122. Clark M, Gray M, Mooney J. New graduate occupational therapists perceptions of near-misses and mistakes in the workplace. Int $J$ Health Care Qual Assur 2013;26:564-76.

123. Kagan I, Barnoy S. Organizational safety culture and medical error reporting by Israeli nurses. J Nurs Scholarsh 2013:45:273-80.

124. Joolaee S, Hajibabaee F, Peyrovi H, et al. The relationship between incidence and report of medication errors and working conditions. Int Nurs Rev 2011;58:37-44.

125. Okuyama A, Sasaki M, Kanda K. The relationship between incident reporting by nurses and safety management in hospitals. Qual Manag Health Care 2010;19:164-72.

126. Albolino S, Tartaglia R, Bellandi T, et al. Patient safety and incident reporting: survey of Italian healthcare workers. Qual Saf Health Care 2010;19(Suppl 3):i8-12.

127. Rasmussen K, Pedersen AH, Pape L, et al. Work environment influences adverse events in an emergency department. Dan Med $J$ 2014;61:A4812.

128. Ballangrud R, Hedelin B, Hall-Lord ML. Nurses' perceptions of patient safety climate in intensive care units: a cross-sectional study. Intensive Crit Care Nurs 2012;28:344-54.

129. Mustafa GR, Rasheed S, Aziz MT. Adverse drug reaction reporting system at different hospitals of Lahore, Pakistan - An evaluation and patient outcome analysis. J App Pharm 2013;4:713-9.

130. Nilsen P. Making sense of implementation theories, models and frameworks. Implement Sci 2015;10:53. 